\title{
Erlang Capacity Analysis of Multi-access Systems Supporting Voice and Data Services
}

\author{
Insoo Koo ${ }^{1}$, Anders Furuskar ${ }^{2}$, Jens Zander ${ }^{2}$, and Kiseon Kim ${ }^{1}$ \\ 1 Dept. of Infor. and Comm., Gwang-Ju Institute of Science and Technology, \\ 1 Oryong-dong, Puk-gu, Gwangju, 500-712, Korea \\ \{kiss and kskim\}@kjist.ac.kr \\ 2 Radio Communication Systems, S3 \\ Isafjordsgatan 30B, KISTA, Stockholm, Sweden. \\ anders.furuskar@ericsson.com, and jens.zander@radio.kth.se
}

\begin{abstract}
In this paper, we analyze and compare the Erlang capacity of multi-access systems supporting several different radio access technologies according to two different operation methods: separate and common operation methods. In the common operation, any terminal can connect to any sub-system while each terminal in the separate operation only can connect to its designated sub-system. In a numerical example with GSM/EDGE-like and WCDMA-like sub-systems, it is shown that we can get up to $60 \%$ Erlang capacity improvement through the common operation method when using a near optimum so-called service-based user assignment scheme. Even with the worst-case assignment scheme, we can still get about $15 \%$ capacity improvement over the separate operation method.
\end{abstract}

\section{Introduction}

Future mobile networks will consist of several distinct radio access technologies, such as WCDMA or GSM/EDGE, where each radio access technology is denoted as "sub-system." Such future wireless networks demanding utilizing the cooperative use of a multitude of sub-systems are named multi-access systems. In the first phase of such multi-access systems, the radio resource management of sub-systems may be performed in a separate way to improve the performance of individual systems independently, mainly due to the fact that the sub-systems have no information of the situation in other sub-systems, or that the terminals do not have multi-mode capabilities. Under such a separate operation method, an access attempt is only accepted by its designated sub-system if possible, and otherwise rejected.

Intuitively, improvement of multiple-access systems is expected in a form of common resource management where the transceiver equipment of the mobile stations supports multi-mode operations such that any terminal can connect to any sub-system. This may be accomplished either through parallel tranceivers in hardware, or using software radio [1]. The common radio resource management functions may be implemented in existing system nodes, but inter-radio access 
technology signaling mechanisms need to be introduced. In order to estimate the benefit of such common resource management of the multi-access systems, some studies are necessary especially in aspects of quantifying the associated Erlang capacity.

As an example of improving the performances of common resource management, for single-service scenarios, the "trunking gain" of multi-access system capacity enabled by the larger resource pool from common resource management has previously been evaluated in [2] through simulation, and multi-service allocation is not considered. In multi-service scenarios, it is expected that the capacity of multi-access systems also depends on how users of different services are assigned on to sub-systems. The gain that can be obtained through the employed assignment scheme can be named as "assignment gain", and further the capacity gain achievable with different user assignment principles has been estimated in 38. These studies however disregard trunking gains. In this paper, we combine both trunking and assignment gains, and quantify the Erlang capacity of the multi-access system. Further, we provide the upper and lower bounds of the Erlang capacity of the multi-access system by considering two extreme cases; one is that all terminals can not support multi-mode function, and the other one is that all terminals can do, which corresponds to the separate and common operations of multi-access systems. In the case of the common operation, we also consider two kinds of user assignment schemes; service-based user assignment [3] as the best case, which roughly speaking assigns users to the sub-system where their service is most efficiently handled, and the rule opposite to the service-based assignment as the worst case reference. With the consideration of these extreme cases, the Erlang capacity of multi-access systems would be a useful guideline for operators of multi-access systems.

\section{Erlang Capacity Analysis of the Multi-access Systems}

In this section, we consider a multi-access system consisting of two sub-systems supporting two services, voice and data, and analyze the Erlang capacity of the multi-access systems according to two different operation methods; separate and common operations. However, it is noteworthy that generalization of this analysis to a case with arbitrary number of sub-systems and services is straightforward, but for lack of space reasons left out.

\subsection{Separate Operation of the Multi-access Systems}

In the separate operation method of the multi-access systems, an access attempt is accepted by its designated sub-system if possible, and otherwise rejected. In order to evaluate the performance of the separate operation in the aspects of traffic analysis, at first we need to identify the admissible region of voice and data service groups in each sub-system. Let $Q_{v}^{l}$ and $Q_{d}^{l}$ be the link qualities such as frame error rate that individual voice and data users experience in the subsystem $l(l=1,2)$ respectively, and $Q_{v, \text { min }}$ and $Q_{d, \text { min }}$ be a set of minimum link 
quality level of each service. Then, for a certain set of system parameters such as service quality requirements, link propagation model and system assumption, the admissible region of the sub-system $l$ with respect to the simultaneous number of users satisfying service quality requirements in the sense of statistic, $S_{s u b, l}$ can be defined as

$$
\begin{aligned}
& S_{s u b, l} \\
= & \left\{\left(n_{(v, l)}, n_{(d, l)}\right) \mid P_{r}\left(Q_{v}^{l}\left(n_{(v, l)}, n_{(d, l)}\right) \geq Q_{v, \text { min }}\right. \text { and }\right. \\
\quad & \left.\left.Q_{d}^{l}\left(n_{(v, l)}, n_{(d, l)}\right) \geq Q_{d, \min }\right) \geq \beta \%\right\} \\
= & \left\{\left(n_{(v, l)}, n_{(d, l)}\right) \mid 0 \leq f_{l}\left(n_{(v, l)}, n_{(d, l)}\right) \leq 1 \text { and } n_{(v, l)}, n_{(d, l)} \in Z_{+}\right\} \text {for } l=1,2
\end{aligned}
$$

where $n_{(v, l)}$ and $n_{(d, l)}$ are the admitted number of calls of voice and data service groups in the sub-system $l$ respectively, $\beta \%$ is the system reliability defined as minimum requirement on the probability that the link quality of the current users in the sub-system $l$ is larger than the minimum link quality level, which is usually given between $95 \%$ and $99 \%$, and $f_{l}\left(n_{(v, l)}, n_{(d, l)}\right)$ is the normalized capacity equation of the sub-system $l$. In the case of linear capacity region, for example $f_{l}\left(n_{(v, l)}, n_{(d, l)}\right)$ can be given as $f_{l}\left(n_{(v, l)}, n_{(d, l)}\right)=a_{l v} \cdot n_{(v, l)}+a_{l d} \cdot n_{(d, l)}$ for $l=1,2$. Such linear bounds on the total number of users of each class, which can be supported simultaneously while maintaining adequate QoS requirements, are commonly found in the literature for CDMA systems supporting multi-class services 45 . Further provided the network state lies within the admissible region, then the QoS requirement of each user will be satisfied with $\beta \%$ reliability.

In the aspects of network operation, it is of vital importance to set up a suitable policy for the acceptance of an incoming call in order to guarantee a certain quality of service. In general, call admission control (CAC) policies can be divided into two categories: number-based CAC (NCAC) and interferencebased CAC (ICAC) 6]. In the case of ICAC, a BS determines whether a new call is acceptable or not by monitoring the interference on a call-by-call basis while the NCAC utilizes a pre-determined CAC threshold. In this paper, we adopt a NCAC-type CAC since its simplicity with which we can apply general loss network model to the system being considered for the performance analysis, even though the NCAC generally suffers a slight performance degradation over the ICAC [6] and the CAC thresholds for the NCAC should be specifically redesigned in the case of changes in propagation parameters or traffic distributions. That is, a call request is blocked and cleared from the system if its acceptance would move the next state out of the admissible region, delimited by Eqn.(1), otherwise it will be accepted.

In order to focus on the traffic analysis of sub-systems under the CAC policy of our interest, we also consider the standard assumptions on the user arrival and departure processes. That is, we assume that call arrivals from users of class $j$ in the sub-system $l$ are generated as a Poisson process with rate $\lambda_{(j, l)}(j=v, d)$. If a call is accepted then it remains in the cell and sub-system of its origin for an exponentially distributed holding time with mean $1 / \mu_{(j, l)}$ which is independent 
of other holding times and of the arrival processes. Then, the offered traffic load of the $j$-th service group in the sub-system $l$ is defined as $\rho_{(j, l)}=\lambda_{(j, l)} / \mu_{(j, l)}$.

With these assumptions, it is well known from $\mathrm{M} / \mathrm{M} / \mathrm{m}$ queue analysis that for given traffic loads, the equilibrium probability for an admissible state $N_{l}$ (三 $\left.\left(n_{(v, l)}, n_{(d, l)}\right)\right)$ in the sub-system $l, \pi\left(N_{l}\right)$ can have a product form on the the truncated state space defined by the call admission strategy such that it is given by 7]:

$$
\pi\left(N_{l}\right)= \begin{cases}\frac{\rho_{(v, l)}^{n_{(v, l)}} \rho_{(d, l)}^{n}(d, l)}{n_{(v, l)} ! n_{(d, l)} !} / \sum_{N_{l}} \in S_{s u b, l} \frac{\rho_{(v, l)}^{n_{(v, l)}} \rho_{(d, l)}^{n_{(d, l)}}}{n_{(v, l)} ! n_{(d, l)} !}, & N_{l} \in S_{s u b, l} \\ 0 & \text { otherwise. }\end{cases}
$$

Subsequently, the blocking probability for a user of class $j$ in the sub-system $l$ is also simply expressed as

$$
B_{(j, l)}=\sum_{N_{l} \in S_{b l k, l}^{j}} \pi\left(N_{l}\right)
$$

where $S_{b l k, l}^{j}$ is the subset of states in $S_{s u b, l}$ which states must move out of $S_{s u b, l}$ with the addition of one user of class $j$. Here, it is noteworthy that $\pi\left(N_{l}\right)$ and $B_{(j, l)}$ are dependent on the admission region $S_{s u b, l}$, and the traffic loads $\rho_{(j, l)}$. Then, the Erlang capacity of the sub-system $l$, defined as the set of supportable offered traffic load of each service, can be calculated as a function of supportable offered traffic loads of all service groups such that

$$
C_{(\text {Erlang }, l)}=\left\{\left(\rho_{(v, l)}, \rho_{(d, l)}\right) \mid \quad B_{(v, l)} \leq P_{(B, v)_{r e q}} \text { and } B_{(d, l)} \leq P_{(B, d)_{r e q}}\right\}
$$

where $P_{(B, j)_{r e q}}$ is the required call blocking probability of service $j$ and can be considered as GoS requirement.

Finally, the combined Erlang capacity, $C_{\text {Erlang }}$, of the multi-access systems under the separate operation is the sum of those of sub-systems such that

$$
\begin{aligned}
& C_{\text {Erlang }}= \\
& \left\{\left(\rho_{v}, \rho_{d}\right) \mid\left(\rho_{v}, \rho_{d}\right) \equiv \sum_{l=1}^{2}\left(\rho_{(v, l)}, \rho_{(d, l)}\right),\left(\rho_{(v, l)}, \rho_{(d, l)}\right) \in C_{(\text {Erlang }, l)} \text { for } l=1,2\right\}
\end{aligned}
$$

\subsection{Common Operation of the Multi-access Systems}

In the common operation of the multi-access systems, the admissible region of the considered multi-access systems depends on how users of different services are assigned onto the sub-systems. That is, according to the employed user assignment scheme in the common operation, the admissible region of multi-access systems can be one of subset of the following set: 
$S_{\text {system }}=$

$\left\{\left(n_{v}, n_{d}\right) \mid\left(n_{v}, n_{d}\right) \equiv \sum_{l=1}^{2}\left(n_{(v, l)}, n_{(d, l)}\right)\right.$, and $0 \leq f_{l}\left(n_{(v, l)}, n_{(d, l)}\right) \leq 1$ for $\left.l=1,2\right\}$

where $n_{v}$ and $n_{d}$ are the admissible number of users of voice and data in the multi-access system.

In the reference [3], Furuskar discussed principles for allocating multiple services onto different sub-systems in multi-access wireless systems, and further derived the favorable near-optimum sub-system service allocation scheme through simple optimization procedures that maximizes the combined multi-service capacity, which here is named as "service-based assignment algorithm." In order to focus on investigating the Erlang capacity improvement through the common operation of multi-access systems by simultaneously considering the trunking gain and the assignment gain, in this paper we only consider two kinds of user assignment schemes for the common operation of multi-access systems, that is, a service-based assignment algorithm, which was proposed in 3] as a near-optimum user assignment method, and a rule opposite to the service-based assignment algorithm as a worst case assignment method. It is noteworthy that the rule opposite to service-based assignment is not likely to be sued in reality but here we adopt it as a interesting reference for the worst case of the common operation method.

In the service-based assignment algorithm, we assign users into the subsystem where their expected relative resource cost for the bearer service type in question is the smallest. That is, when a user with service type $j$ is coming in the multi-access system $(j=v$ or $d)$, then we assign the user to the sub-system $\hat{l}$ that meets the following.

$$
\hat{l}=\arg \left\{\min _{l}\left(\frac{\partial f_{l}\left(n_{(v, l)}, n_{(d, l)}\right)}{\partial n_{(j, l)}} / \frac{\partial f_{l}\left(n_{(v, l)}, n_{(d, l)}\right)}{\partial n_{(\sim j, l)}}\right)\right\}
$$

where $\sim j$ is the 'other service' that is, if $j=v$ then, $\sim j$ is $d$. For the case that each sub-system has a linear capacity region, then the assignment rule can be simply expressed as $\hat{l}=\arg \left\{\min \left(\frac{a_{l j}}{a_{l \sim j}}\right)\right\}$.

On the other hand, in the rule opposite to the service-based assignment algorithm, we assign the user having service type $j$ to the sub-system $\hat{l}$ that meets the following.

$$
\hat{l}=\arg \left\{\max _{l}\left(\frac{\partial f_{l}\left(n_{(v, l)}, n_{(d, l)}\right)}{\partial n_{(j, l)}} / \frac{\partial f_{l}\left(n_{(v, l)}, n_{(d, l)}\right)}{\partial n_{(\sim j, l)}}\right)\right\}
$$

According to the employed user assignment scheme, we can obtain corresponding admissible region of the multi-access systems under the common operation. If we denote $S_{s-b a s e d}$ as the admissible region of the multi-access systems with the service-based assignment scheme, and $S_{o p p-s-b a s e d}$ as one with the rule 


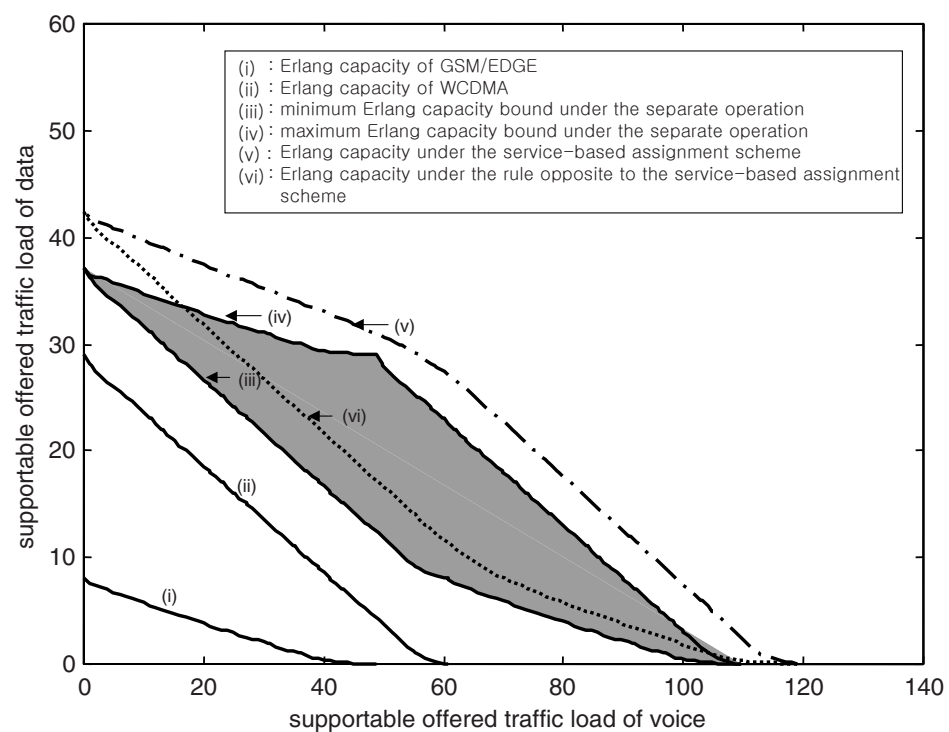

Fig. 1. Erlang capacity of a GSM/EDGE-like and WCDMA-like multi-access system.

opposite to the service-based assignment scheme, respectively. Then, we can calculate the corresponding Erlang capacities for above two assignment scheme using the similar method presented in previous section, by using Eqns(2-4) after replacing $S_{s u b, l}$ with $S_{s-b a s e d}$ and $S_{o p p-s-b a s e d}$, respectively.

\section{$3 \quad$ Numerical Results}

So far we have presented analytical procedures to investigate the Erlang capacity of multi-access systems. In this section, we will consider two set of scenarios with different bearer capabilities and quality requirements of sub-systems to visualize the capacity gains of multi-access system according to the two operation methods. First scenario is a case with coexisting GSM/EDGE-like and WCDMAlike sub-systems as a practical example. The other is more artificial case to consider the effect of sub-system capacities on the Erlang capacity of multiaccess systems.

As a practical example, let's firstly consider a case, $P=2$ with coexisting GSM/EDGE-like and WCDMA-like sub-systems. When a spectrum allocation of $5 \mathrm{MHz}$ is assumed for both systems, admissible capacity regions of both systems supporting mixed voice and data traffics are modeled as a linear region such that $f_{l}\left(n_{(v, l)}, n_{(d, l)}\right)$ is given as $a_{l v} \cdot n_{(v, l)}+a_{l d} \cdot n_{(d, l)}$ for $l=1,2$ where GSM/EDGE-like system is denoted as sub-system 1 while WCDMA-like system as sub-system 2. Further, $\left(a_{1 v}, a_{1 d}\right)$ and $\left(a_{2 v} a_{2 d}\right)$ are given as $(1 / 621 / 15)$ and $(1 / 751 / 40)$ respectively, for standard WCDMA and EDGE data bearers and a circuit switched 
equivalent (CSE) bitrate requirement of $150 \mathrm{Kbps}$ [8]. Fig. 1] shows the resulting Erlang capacity regions when the required call blocking probability is set to $1 \%$. Fig. I(i) and (ii) show the Erlang capacity of GSM/EDGE and WCDMA, respectively. Then, the Erlang capacity of multi-access system under the separate operation can be given as the vector sum of those of sub-systems as like Fig. 1 It is noteworthy that the Erlang capacity line, stipulating the Erlang capacity region of multi-access system, depends on the service mix in the sub-systems, and lies between the minimum bound line (Fig. T(iii)) and the maximum bound line (Fig. 1(iv)). This means that the shadowed traffic area, delimited by Fig. 1 (iii) and Fig. 11iv), is not always supported by the multi-access system under the separate operation. For example, the traffic load of $(46,29)$ can be supported only when the GSM/EDGE supports the voice traffic of 46, and the WCDMA supports the data traffic of 29 , but this occasion is very rare. Subsequently, we should operate the system with the Erlang capacity region stipulated by Fig. 1 (iii) for the sake of stable system operation. On the other hand, Fig. 11(v) shows the Erlang capacity region of multi-access system under the service-based assignment algorithm. In this case, with the service-based assignment scheme we assign voice users to GSM/EDGE as far as possible, and data users to WCDMA since GSM/EDGE is relatively better at handling voice users than WCDMA, and vice-versa for data users. As a result, it is observed that we can get about 95\% capacity improvement through the service-based assignment algorithm over the separate operation where we utilize the supportable area of Erlang capacity for the performance comparison. Fig. 1(vi) also shows the Erlang capacity region of multi-access system when assigning users according to the rule opposite to the service-based assignment algorithm. In this case, the voice users are as far as possible assigned to WCDMA and as many data users as possible to GSM/EDGE, which corresponds to the worst-case scenario in the common operation. The resulting Erlang capacity is dramatically lower than that of the service-based assignment algorithm (roughly 54\% capacity reduction). However, ever in the worst case, we know that the Erlang capacity of multi-access system under the common operation still can provide about $27 \%$ capacity improvement over the separate operation.

Secondly, we consider an artificial case to consider the effect of air-link capacities of sub-systems on the Erlang capacity of multi-access systems where the admissible regions of each sub-system are also delimited by the linear bound, and $\left(a_{1 v} a_{1 d}\right)$ and $\left(a_{2 v} a_{2 d}\right)$ are given as $(1 / 101 / 10)$ and $(1 / 201 / 10)$, respectively. Fig. 2 shows the resulting Erlang capacity regions for the two operation methods. With the service-based assignment scheme, in this case we assign voice users to sub-system 2 as far as possible, and data users to sub-system 1 since sub-system 2 is relatively better at handling voice users than sub-system 1 , and vice-versa for data users. As a result, we can achieve a gain of up to $37 \%$ over the rule opposite to the service-based assignment through the service-based user assignment, and the gain of up to $88.5 \%$ over the separate operation method. When comparing these results with those of the previous example, we also know that the Erlang capacity gains of multi-access systems, which can be achieved 


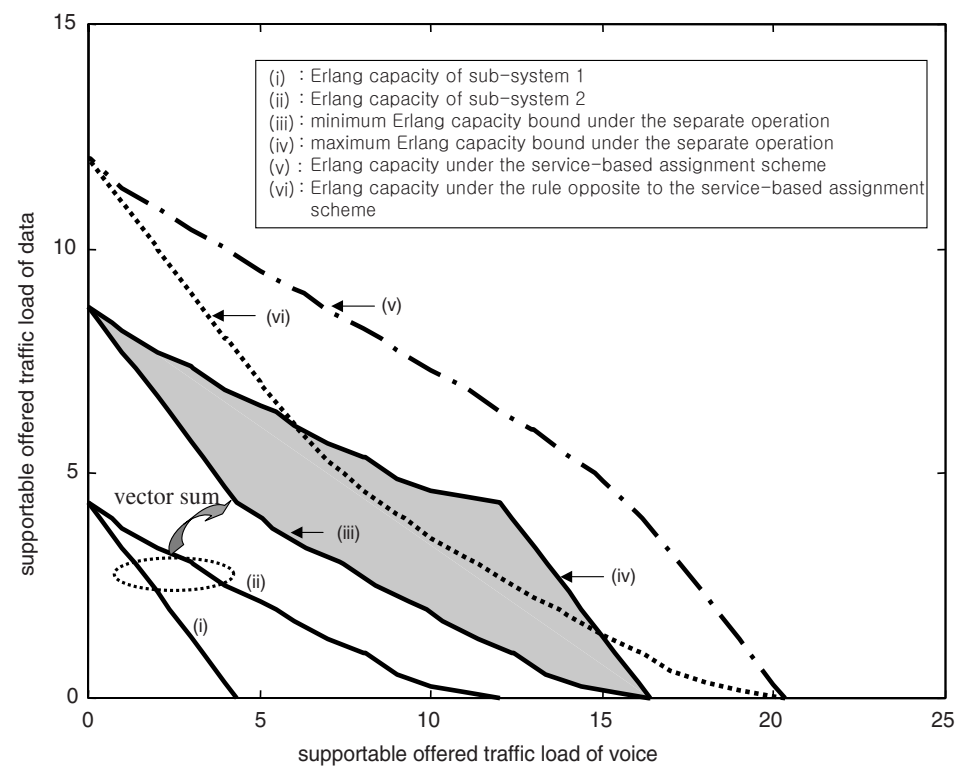

Fig. 2. Erlang capacity of a multi-access system for the two operation methods.

by the operation methods, are very sensitive to the sub-system capacities such as the shape and the area of the capacity.

Finally, Fig. 3 shows the Erlang capacity gain of a multi-access system according to the traffic mix-ratio between voice and data for the previous two numerical examples. Here, we define the traffic mix-ratio as $\rho_{v} /\left(\rho_{v}+\rho_{d}\right)$, and use the total supportable traffic load of the system for the performance comparison, i.e the sum of the maximum supportable voice and data traffic for a certain service-mix ratio. Noting that the Erlang improvement of common mode operation over the separate operation converges into a trunking gain as the traffic mix-ratio between voice and data goes to 0 or 1, we know that the Erlang improvement of common mode operation is mainly due to the trunking efficiency gain when the rule opposite to the service-based assignment scheme is used, and that the gain is less sensitive to the traffic mix-ratio between voice and data while it is sensitive to the sub-system capacities. On the other hand, Fig. 1 shows that the Erlang capacity improvement in the case of the service-based assignment scheme varies according to the traffic mix-ratio between voice and data. This means that in this case we can get both a trunking efficiency gain and a service-based assignment gain simultaneously. It is noteworthy that the trunking efficiency gain is rather insensitive to the service mix, whereas the service-based assignment gain depends significantly on the service mix. The service-based assignment scheme is thus more beneficial in mixed service scenarios.

This paper can be further extended, by considering different service control mechanisms, and the capabilities that each terminal can handle multi-mode 


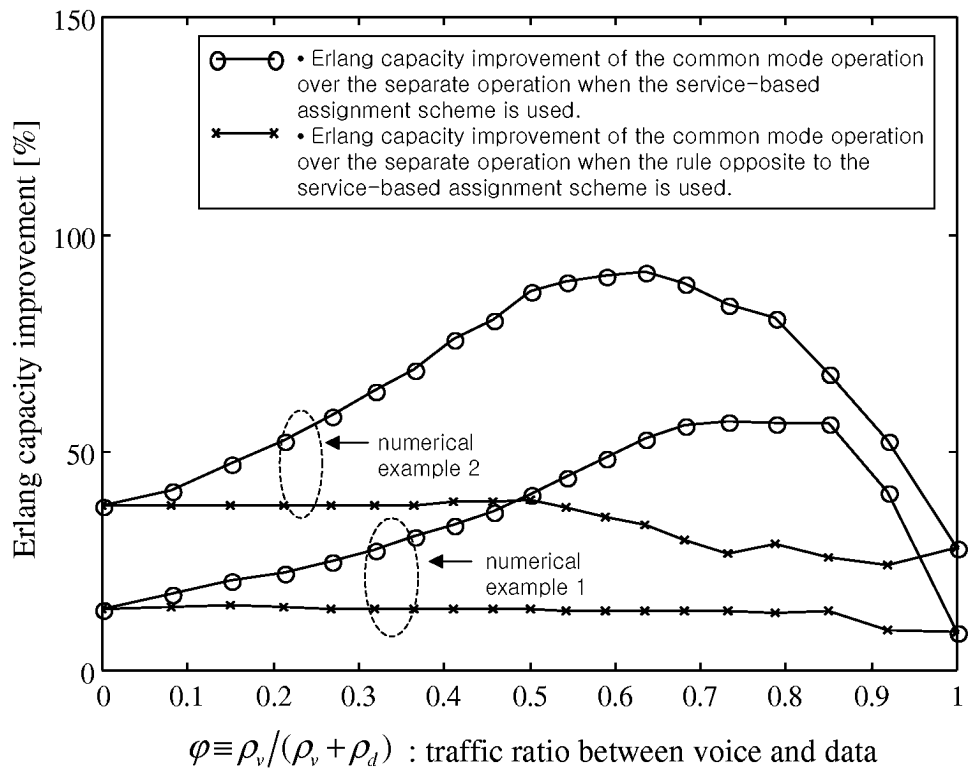

Fig. 3. Erlang capacity improvement of a multi-access system according to the traffic mix-ratio between voice and data, $\varphi$

function. For the lack of space, in this paper we have only dealt with two extreme cases; separate and common operations of multi-access systems. For the case that non-multi-mode and multi-mode terminals coexist, however we can expect that the corresponding Erlang capacity will fall into the regions between the Erlang capacity under the separate operation and that under the common operation, even though we can not exactly mention how and how much the fraction of non-multi-mode terminals affects overall performance of multi-access system. The corresponding Erlang capacities according to different service control mechanisms will also exist in the regions between the Erlang capacity under the service-based user assignment and that under the rule opposite to the servicebased assignment, since the service-based user assignment is proved to be the optimum sub-system service allocation scheme [3].

\section{Conclusion}

In this paper, we investigate the Erlang capacity of multi-access systems according to two different operation methods: separate and common operation methods. Through numerical examples, we observe that the Erlang capacity improvement that can be obtained through common operation method is two-fold. First, a trunking efficiency gain is achieved due to the combining of resource pools. This gain depends on the sub-system capacities, for small sub-system capacities the gain is significant. Secondly, a service-based assignment gain can 
be achieved by assigning users to the sub-system where their service is most efficiently handled. This gain depends on the shape of the sub-system capacity regions. Roughly, the more different these are, the larger the gain. It is also observed that the trunking efficiency gain is rather insensitive to the service mix, whereas the service-based assignment gain depends significantly on the service mix. Finally, the results of the paper are expected to be used as a guideline to operate and dimension future multi-access systems.

Acknowledgement. This work was supported by Korea Science and Engineering Foundation (KOSEF) through the UFON research center at Gwangju Institute of Science and Technology. Insoo Koo particularly was supported by the Basic Research Program of the KOSEF.

\section{References}

1. S. Ogose, "Application of software radio to the third generation mobile telecommunications," pp.1212-1216, Proc. of VTC, 1999.

2. A. Tolli, P. Hakalin, and H. Holma, "Performance evaluation of common radio resource management (CRRM)," pp.3429- 3433, Proc. of ICC, 2002.

3. A. Furuskar, "Allocation of multiple services in multi-access wireless systems," pp.261-265, Proc. of MWCN, 2002.

4. J. Yang, Y. Choi, J. Ahn and K. Kim, "Capacity plane of CDMA system for multimedia traffic," IEE Electronics Letters, vol.33, no.17, pp.1432-1433, Aug. 1997.

5. A. Sampath, P. Kumar and J. Holtzman, "Power control and resource management for a multimedia CDMA wireless system," pp.21-25, Proc. of PIMRC, 1995.

6. Y. Ishikawa and N. Umeda, "Capacity design and performance of call admission cotnrol in cellular CDMA systems," IEEE JSAC, vol. 15, pp.1627-1635, Oct. 1997.

7. F. Kelly, "Loss networks," The Annuals of Applied Probability, Vol.1, pp.319-378, 1999.

8. A. Furuskar, "Radio resource sharing and bearer service allocation for multi-bear service, multi-access wireless networks," Ph.D thesis, available at http://www.s3.kth.se/radio/Publication/Pub2003/af_phd_thesis_A.pdf 